\title{
Overcoming Drug Resistance in Pancreatic Cancer
}

\author{
Jiang Long ${ }^{1,2}$, Yuqing Zhang ${ }^{1}$, Xianjun $\mathrm{Yu}^{2}$, Jingxuan Yang ${ }^{1,3}$, Drake LeBrun ${ }^{3}$, Changyi \\ Chen ${ }^{1}$, Qizhi Yao ${ }^{1}$, and Min Li ${ }^{1,3,}{ }^{*}$ \\ ${ }^{1}$ Molecular Surgeon Research Center, Elkins Pancreas Center, Michael E. DeBakey Department \\ of Surgery, Baylor College of Medicine, Houston, Texas 77030, USA \\ ${ }^{2}$ Department of Pancreas \& Hepatobiliary Surgery, Pancreatic Cancer Center/Institute, Cancer \\ Hospital, Shanghai Medical College, Fudan University, 270 Dong'an Road, Shanghai, 200032, \\ People's Republic of China \\ ${ }^{3}$ The Vivian L. Smith Department of Neurosurgery, the University of Texas Health Science Center \\ at Houston, Medical School, Houston, Texas 77030, USA
}

\begin{abstract}
Introduction-Pancreatic cancer has the worst survival rate of all cancers. The current standard care for metastatic pancreatic cancer is gemcitabine, however, the success of this treatment is poor and overall survival has not improved for decades. Drug resistance (both intrinsic and acquired) is thought to be a major reason for the limited benefit of most pancreatic cancer therapies.
\end{abstract}

\begin{abstract}
Areas covered-Previous studies have indicated various mechanisms of drug resistance in pancreatic cancer, including changes in individual genes or signaling pathways, the influence of the tumor microenvironment, and the presence of highly resistant stem cells. This review summarizes recent advances in the mechanisms of drug resistance in pancreatic cancer, and potential strategies to overcome this.
\end{abstract}

Expert Opinion-Increasing drug delivery efficiency and decreasing drug resistance is the current aim in pancreatic cancer treatment, and will also benefit the treatment of other cancers. Understanding the molecular and cellular basis of drug resistance in pancreatic cancer will lead to the development of novel therapeutic strategies with the potential to sensitize pancreatic cancer to chemotherapy, and to increase the efficacy of current treatments in a wide variety of human cancers.

\section{Keywords}

Drug resistance; pancreatic cancer

\section{Introduction}

Pancreatic cancer is one of the most difficult human cancers to treat due to the inability to detect disease at an early stage and the lack of effective therapies. Although there has been some progress in the use of improved diagnostic methods and development of novel targeted therapies, the overall survival rate has not improved over the last decade. Pancreatic cancer remains the fourth leading cause of cancer death in the United States, with an annual

\footnotetext{
*Address correspondence to: Min Li, PhD, The Vivian L. Smith Department of Neurosurgery, The University of Texas Health Science Center at Houston, Medical School, 6431 Fannin Street, MSB 3.000, Houston, TX 77030, Tel: (713) 500-6491, Min.Li@uth.tmc.edu. Declaration of interest

The authors declare no conflict of interest.
} 
mortality nearly equivalent to the annual incidence 1-4. Eighty percent of pancreatic adenocarcinomas are unresectable in patients with clinical symptoms 4,5 . Clearly, there is a need to understand more about the molecular mechanisms of pancreatic cancer pathogenesis and to develop effective treatments for pancreatic cancer. Important information can be gained by studying the progressive development of precursor lesions and invasive cancers in animal models of pancreatic cancer. Genomic profile studies, such as serial analysis of gene expression (SAGE), microarray analysis, and DNA sequencing have also been used to identify genes overexpressed in pancreatic cancer and to detect genetic mutations in oncogenes and tumor suppressor genes 6-10. The identification of these overexpressed genes might lead to development of new markers and screening techniques as well as new gene therapy strategies. However, many of these genes are non-specific in pancreatic cancer, and the targeted therapies are ineffective. Therefore, in order to improve diagnosis and therapeutics of pancreatic cancer, it is critical to target genes which are either more specific in pancreatic cancer, or genes which are involved in multiple pathways, and to enhance the efficacy of the current chemo- and radiation therapy for pancreatic cancer.

One of the important features of pancreatic cancer is the high resistance to traditional chemo- and radiation therapy, including both intrinsic (de novo) and acquired (therapyinduced) chemoresistant behaviors of the cancer cells. Patients with pancreatic cancer usually present with locally advanced, unresectable or metastatic diseases. Even for patients with resectable disease, most of them will relapse. Therefore, postoperative chemo- or radiation therapy is still necessary for pancreatic cancer management although there is argument that preoperative therapy (neoadjuvant treatment) works better than the postoperative therapy, and other people think chemo- and radiation therapy is not necessary at all.

The most commonly used chemotherapy for pancreatic cancer, gemcitabine, has modest clinical benefit and may not improve overall survival to a clinically meaningful degree. The lack of significant clinical response of pancreatic cancer patients to chemotherapy is likely due to the inherent chemoresistance of pancreatic cancer cells as well as impaired drug delivery pathways. Understanding the underlying mechanisms of drug resistance in pancreatic cancer is critical to develop new effective treatments for this deadly disease.

Previous studies have indicated various mechanisms of drug resistance in pancreatic cancer, including changes in individual genes or signaling pathways, the influence of the tumor microenvironment, and the presence of highly resistant stem cells. This review summarizes recent advances in the mechanisms of drug resistance in pancreatic cancer and potential strategies to overcome drug resistance.

\section{Pancreatic cancer is highly resistant to chemotherapy drugs}

Gemcitabine was approved by FDA in 1997 as the first-line chemotherapy drug for patients with locally advanced or metastatic pancreatic adenocarcinoma. Initially 126 patients were enrolled in this randomized phase III clinical trial 11, the gemcitabine group showed better clinical outcomes with increased median survival compared with the 5-fluorouracil (5-FU) group. Clinical benefit response was $23.8 \%$ of gemcitabine-treated patients compared with only $4.8 \%$ of 5 -FU-treated patients $(\mathrm{P}=0.0022)$. The median survival durations were 5.65 and 4.41 months for gemcitabine-treated and 5-FU-treated patients, respectively $(\mathrm{P}=0.0025)$. The survival rate at one year was $18 \%$ for gemcitabine patients and $2 \%$ for 5-FU patients 11 . Over the past decades many efforts have been made to improve the clinical efficacy of chemotherapy. Up to date, gemcitabine still remains the standard treatment for pancreatic cancer patients. Combinational trials with gemcitabine have been reported which include cytotoxic agents (5-FU, cisplatin, oxaliplatin, and capecitabine) 12-15, and biological agents 
(erlotinib, Cetuximab, and bevacizumab) 16-18. Some of those trials demonstrated higher clinical benefits and relatively longer survival; however none of those combination regimens have proven to be significantly more effective than gemcitabine alone as the first-line therapy. The overall survival rate remains unchanged.

Impaired drug delivery pathways have been shown to be another reason for the drug resistance in pancreatic cancer 19. It is believed that within pancreatic tumors there are solid tumor masses which lack adequate vasculature with intensive desmoplastic reaction 20. Therefore it is difficult for any drug to penetrate the stroma area and reach the inside of the solid tumors. It is also suggested that chemotherapy can further activate the drug resistant pathway in pancreatic cancer.

\section{Understanding the mechanisms of drug resistance in pancreatic cancer}

Previous studies have suggested a variety of mechanisms of drug resistance in pancreatic cancer, including aberrant gene expression, mutations, deregulation of key signaling pathways (such as NF- $\mathrm{kB}, \mathrm{Akt}$, and apoptosis pathways), epithelial-mesenchymal transition (EMT) and the presence of stroma cells, highly resistant cells and stem cells. Each of those mechanisms contributes to drug resistance in pancreatic cancer from different aspects, and suggests different therapeutic targets (Figure 1).

\section{Aberrant Gene expression}

Genetic and/or epigenetic alterations have been associated with gemcitabine resistance in pancreatic cancer. Multidrug resistance (MDR) causes resistance to many therapeutic agents, and leads to ineffectiveness in chemotherapeutic management of pancreatic cancer 21. The MDR phenotype is often attributed to the overexpression of certain drug efflux pumps, which leads to decreased intracellular drug accumulation. Members of the ATPbinding cassette $(\mathrm{ABC})$ transporter superfamily have been shown to be key mediators of drug efflux and multidrug resistance in many types of tumors 22-24. ABC transporters are thought to operate as hydrophibic vacuum cleaners by expelling non-polar compounds from the plasma membrane using energy released from ATP hydrolysis 25 . To date, 49 human $\mathrm{ABC}$ transporters have been identified and categorized into seven subfamilies 26. In particular, the ABC-B, -C, and -G subfamilies have been shown to be significant contributors to multidrug resistance in tumor cell lines 23, 27. P-glycoprotein is one of the best characterized $\mathrm{ABC}$ transporters. Overexpression of P-glycoprotein is associated with poor prognosis of many human cancers, and contributes to drug resistance 21. Other $A B C$ transporters, such as MRP1 (ABCC1), MRP3 (ABCC3), MRP4 (ABCC4), and MRP5 (ABCC5), have been implicated in drug resistance in pancreatic tumors 28, 29 and been shown to confer resistance to pancreatic cell lines against common chemotherapeutic drugs including gemcitabine 30 and 5-fluorouracil 31. Due to the key roles played by ABC transporters in multidrug resistance, inhibitors of $A B C$ transporters may lead to enhanced efficacy of chemotherapeutic strategies targeting pancreatic cancer. The intracellular uptake of gemcitabine is dependent on nucleoside transporters, such as human equilibrate nucleoside transporter-1 (hENT1) 32. In vitro studies have shown that decrease in hENT1 expression leads to limited intracellular uptake of gemcitabine 33 .

Many enzymes are involved in the metabolism of gemcitabine, or are targets for the different metabolites of gemcitabine. Therefore, any changes in enzyme activity can also influence the sensitivity to gemcitabine 34 . For example, deoxycytidine kinase (DCK) and ribonucleoside reductases $\mathrm{M} 1$ and $\mathrm{M} 2$ have been shown to correlate with gemcitabine resistance 35. Copper transporter 1 (hCTR1), which controls cisplatin accumulation in mammalian cells, is key to cisplatin drug resistance 36. Aberrant expression of genes associated with cellular survival and apoptosis, such as the S100 family member S100A4, 
survivin, plk1, snail, and SphK1 is thought to be important in drug resistance; increased expression and activation of c-Src and c-Met have also been implicated in gemcitabine resistance in pancreatic cancer (Fig. 1.) 35, 37-41.

\section{Genetic Mutations}

Drug resistance in many cancers has been attributed to genetic mutations in key genes. It has been shown that BRCA1- or BRCA2-deficient cells are very sensitive to DNA cross-linking agents, and tumors holding BRCA1 and BRCA2 mutations show enhanced sensitivity to carboplatin 42. Recent studies have indicated that BRCA1 or BRCA2 deficiency sensitizes cells to the treatment of inhibitors of the enzyme poly(ADP-ribose) polymerase (PARP), such as KU0058948 43, 44. Based on the importance of those two genes in cancers, the synthetic lethality therapy holds great promise, in which targeting one key gene in a cancer where the other one is defective is selectively lethal to the tumor cells but less toxic to the normal cells. However, this sensitivity can be reversed by mutations, and resistance to PARP inhibition can be acquired by deletion of a mutation in BRCA2 gene as shown in pancreatic cancer cells Capan-1 45. This gain-of-function mutation in BRCA2 gene might represent a new mechanism for drug resistance in pancreatic cancer and other cancers. A similar mechanism was also seen for carboplatin resistance in ovarian cancer in which BRCA2 gene was mutated 46.

\section{Deregulations of key signaling pathways}

In addition to aberrant gene expressions, activation or inhibition of transcription factors and key signaling pathways also contribute to drug resistance. Many signal pathways have been indicated to be involved in drug resistance in pancreatic cancer, such as nuclear factor $\mathrm{kB}$ (NF- $\kappa \mathrm{B})$, Akt and Notch pathways. Resistance to chemotherapy in pancreatic cancer is largely associated with constitutively activated NF- $\kappa \mathrm{B}$, which is further activated by chemotherapeutic drugs and gamma irradiation.

$\mathrm{NF}-\kappa \mathrm{B}$ is a key nuclear transcription factor that is crucial for the regulation of apoptosis, inflammation, tumorigenesis and various autoimmune diseases by regulating expression of a large number of genes 47. A recent study found that 3,3-diindolylmethane (DIM), a natural compound from cruciferous vegetables, potentiates the killing of pancreatic cancer cells in vivo and in vitro by downregulation of constitutive as well as drug-induced activation of NF- $\mathrm{kB}$ and its downstream target genes such as survivin, Bcl-xL, XIAP and cIAP. In an orthotopic animal model, tumor size was significantly reduced when DIM was given in combination with oxaliplatin compared with the monotherapy. Further analysis indicated that DIM treatment caused loss of phospho-p65 and downregulation of NF- $\mathrm{kB}$ activity and its downstream genes in those tumors. These results suggest that DIM could abrogate chemotherapeutic drug (cisplatin, gemcitabine, and/or oxaliplatin)-induced activation of NF$\kappa \mathrm{B}$, resulting in the chemosensitization of pancreatic tumors to conventional therapies 48 , 49.

Alterations of the phosphatidylinositol 3-kinase (PI3K)/Akt signaling pathway in human cancers have been found and are thought to be mediators of chemoresistance to gemcitabine inpancreatic cancer 32, 35, 50-52. Ng et al showed increased gemcitabine-induced apoptosis in PK1 and PK8 human pancreatic cancer cells after treatment with wortmannin and LY294002, which inhibit the PI3K-protein kinase B (PKB)/Akt cell survival pathway, suggesting therapeutic potentials of PI3K inhibitors in increasing chemosensitivity to gemcitabine in pancreatic cancer 51. Recently Liau et al reported that Akt was a key downstream target of HMGA1-dependent signaling in pancreatic cancer and they have demonstrated that HMGA1 promoted chemoresistance to gemcitabine through an Aktdependent mechanism in a nude mouse orthotopic xenograft model 50 . These findings 
suggested that targeted suppression or inactivation of HMGA1 could be a potential therapeutic strategy for reversing drug resistance to gemcitabine in pancreatic cancer.

\section{EMT and Chemoresistance}

Epithelial-mesenchymal transition (EMT) has been implicated as a potential mechanisms for drug resistance. Pancreatic cancer cells with a mesenchymal phenotype are usually gemcitabine-resistant. Ellenrieder et al demonstrated that EMT induced by TGF- $\beta 1$ contributes to pancreatic cancer cell scattering, migration, and invasion in TGF- $\beta 1$ responsive pancreatic cancer cells (Panc-1, Colo-357, and IMIM-PC1) 53. The molecular mechanisms of EMT and cancer progression/chemoresistance are not fully understood. Recent studies have shown that many signaling pathways including the Wnt, TGF- $\beta$, Hedgehog, Notch, and NF- $\mathrm{KB}$ signaling pathways were critical for EMT induction 54-58. Notch signaling pathway is thought to play a major role in the acquisition of EMT and cancer stem-like cell phenotype. The Notch signaling pathway is activated in pancreatic cancer precursors and advanced tumors. It is required for the expansion of undifferentiated pancreatic progenitor cells during embryonic development and has been implicated in the progression of pancreatic cancer 59. Wang et al 54 showed that Notch-2 and its ligand, Jagged-1, were highly upregulated in gemcitabine-resistant pancreatic cancer cells and the downregulation of Notch signaling was associated with decreased invasion of gemcitabineresistant cells. Furthermore, blocking of Notch signaling by siRNA led to partial reversal of the EMT phenotype, resulting in the mesenchymal-epithelial transition, which was associated with decreased expression of vimentin, ZEB1, Snail, Slug, and NF- $\kappa B$. These results suggest that blocking of Notch signaling could be a potential targeted therapy for overcoming chemoresistance of pancreatic cancer 54.

\section{Hypoxia and stroma cells}

One of the unique features of pancreatic cancer is the presence of dense desmoplastic stroma, which is not found in other distal metastatic tumors. The existence of extensive desmoplastic stroma and the hypoxic microenvironment have been reported to play a critical role in pancreatic cancer progression, and significantly affect the penetration and delivery of chemotherapeutic drugs. Recent studies have shown that fibroblasts, which are now recognized as activated pancreatic stellate cells (PSCs) 60, play an important role in the generation of the desmoplastic stroma in pancreatic cancer and affect biological properties of pancreatic cancer cells 61, 62. Hwang et al showed that PSC-conditioned medium increased pancreatic cancer cell proliferation, migration, invasion, and colony formation in a dose-dependent manner, and gemcitabine and radiation therapy were less effective in cancer cells treated with PSC-conditioned medium 61. The in vivo study indicated that PSCs enhanced tumor growth and metastasis. These results provide strong evidence that stromal fibroblasts produce secreted factors to promote pancreatic tumor progression and impair response to chemo- and radiation therapy.

Another mechanism by which the tumor-associated stroma in pancreatic cancer could impair response to chemotherapy is by acting as a barrier to drug delivery. Olive et al demonstrated that depletion of pancreatic tumor stroma by inhibition of the Hedgehog signaling pathway stimulated angiogenesis and consequently augmented the delivery and efficacy of gemcitabine in a genetically-engineered mouse model 63 . These results suggest that impaired drug delivery by stroma and hypoxia may be another reason for chemoresistance in pancreatic cancer 63.

\section{Cancer stem cells}

The presence of cancer stem cells (CSCs) may also contribute to pancreatic cancer's resistance to chemotherapy and the high recurrence rate after clinical remission 64, 65. Li et 
al first reported the identification of human pancreatic cancer stem cells, which is defined by the expression of cell surface markers CD44+CD24+epithelial specific antigen (ESA)+. Cells expressing these markers were highly tumorigenic and had the ability to both selfrenew and produce differentiated progeny, resembling the patient's primary tumors 66 . The developmental signaling molecules sonic hedgehog and Bmi-1 were found to be upregulated in pancreatic cancer stem cells. Recently, CD133 was also identified on the surface of fast proliferative primary pancreatic cancers and pancreatic cancer cell lines 67 , and has been previously used to identify a cancer stem cell population in brain and colon cancers 68,69 , indicating that CD133 is a another potential marker for pancreatic cancer stem cells. Recent studies also suggest that Aldehyde Dehydrogenase (ALDH) activity is another marker for pancreatic cancer stem cell population 70, 71. Discovery of a more specific and complete set of cell surface markers may help to further characterize pancreatic cancer stem cells, and develop effective treatments targeting pancreatic cancer stem cells.

Shah et al have observed that gemcitabine-resistant pancreatic cancer cells have increased expression of stem cell markers CD24, CD44 and ESA 57. They also showed that the resistant cancer cells possessed the morphologic and biochemical properties of EMT. They further demonstrated a link between c-Met activation, gemcitabine resistance, tumor aggressiveness, cancer stem cells, and EMT. In addition, it has been shown that treatment with ionizing radiation and gemcitabine results in the enrichment of the CD44+CD24+ESA + cell population in human primary pancreatic cancer xenografts 64,65 . However, although recent studies have shown Wnt/ $\beta$-catenin and Notch signaling may be implicated in the chemoresistance and radioresistance, the exact mechanisms involved in the resistance of pancreatic cancer stem cells to conventional chemotherapy and radiation therapy, and the contribution of developmental pathways to the drug resistance, remains unclear 65 . How to specifically inhibit pancreatic cancer stem cells to overcome drug resistance of pancreatic cancer warrants further investigation. This is a challenging area of research, but successfully eradicating pancreatic cancer stem cells will have a significant clinical impact, and will definitely benefit patients with pancreatic cancer.

\section{Strategies to overcome drug resistance in pancreatic cancer}

Based on the understanding of the drug resistance mechanisms in pancreatic cancer, many studies have been done to develop effective therapies targeting those aberrantly expressed genes, signaling pathways, and microenvironment, which contribute to drug resistance in pancreatic cancer. In combination with standard chemotherapy, those new therapeutic efforts may overcome drug resistance and enhance the efficacy of therapy compared with single agent treatment for pancreatic cancer.

\section{Targets for inhibiting drug resistance}

MDR proteins (MRP1, MRP3, MRP4, MRP5 and P-glycoprotein) are well known drug resistance proteins, and are highly expressed in pancreatic cancer cells. Reversin 121 (R121), a hydrophobic peptide, which binds to purified MDR proteins with high affinity inhibiting substrate extrusion, can sensitize the cells to chemotherapy drugs. Katrin et al demonstrated that R121 restored chemosensitivity and reduced proliferative behavior of pancreatic cancer cells in vitro. In vivo treatment using chemotherapy drugs 5-fluorouracil, gemcitabine and cisplatin in combination with R121 reduced peritoneal, hepatic and pulmonary metastatic spread compared with any of the individual chemotherapeutic drugs alone according to several case reports of pancreatic cancer patients 72 , indicating the therapeutic potential of blocking MDR proteins in decreasing drug resistance in pancreatic cancer treatment. 
Activation of NF- $\mathrm{kB}$ has been shown to induce pancreatic cancer cell proliferation, invasion, angiogenesis, metastasis, and drug resistance. Therefore, specific inhibitors of NF$\kappa \mathrm{B}$ and other molecular therapies targeting NF- $\mathrm{BB}$ pathway may provide potent anti-cancer treatment. Many chemopreventive agents, including curcumin, resveratrol, emodin, green tea polyphenols, silymarin, $\beta$-lapachone, caffeic acid phenethyl ester, and sulindac are known to suppress the activation of NF- $\kappa B$ and NF- $\kappa B$-regulated gene expression 49. Most recently Banerjee et al demonstrated that 3,3'-diindolylmethane (DIM), a natural plantderived product, could induce cell growth inhibition and apoptosis by suppressing NF- $\mathrm{\kappa B}$ and its downstream genes, and abrogate chemotherapeutic drug (cisplatin, gemcitabine, and oxaliplatin)-induced activation of NF- $\mathrm{\kappa B}$ in an orthotopic animal model, resulting in the reversal of chemoresistance in pancreatic cancer 48.

In addition to natural compounds that inhibit NF-kB which may also inhibit off-target molecules, specific targeting of NF-kB using siRNA can improve response to gemcitabine. Pan et al demonstrated that siRNA-mediated inhibition of p65/relA induced apoptosis and increased gemcitabine killing of gemcitabine-sensitive pancreatic cancer cells such as BxPC-3 cells 73. Silencing of p65/relA was found to be effective alone and in combination with gemcitabine in gemcitabine-sensitive but not gemcitabine-resistant pancreatic cancer cells (such as Panc-1 and MIA PaCa-2 cells). Thus, NF- $\mathrm{kB}$ may be a useful therapeutic target for a subset of pancreatic cancers. Those studies not only suggest that blocking NF$\kappa \mathrm{B}$ pathway can decrease drug resistance, but also indicate the heterogeneity of pancreatic cancer. Targeting one single molecule or pathway may not be enough to overcome the drug resistance in pancreatic cancer. Combination therapy targeting multiple pathways may have better efficacy in pancreatic cancer treatment.

The PI3K/Akt signaling pathway is frequently deregulated in pancreatic cancers 74,75 and is activated in pancreatic cancer in response to chemotherapy. Thus, Akt is another attractive therapeutic target as it is only transiently activated in normal cells, but is constitutively activated in cancer cells. Fahy and colleagues examined the effect of Akt inhibition in sensitizing human pancreatic cancer cell lines, MIA PaCa-2, Panc-1 and BxPC-3 cells. They found that only MIA PaCa-2 cells, which had high level of Akt activity, demonstrated increased apoptosis after Akt inhibition in combination with gemcitabine, while Panc-1 or BxPC-3 cells, which had lower levels of basal Akt activity, did not show significantly increased apoptosis with Akt inhibition 74. These results suggest that high basal Akt activity is required for this targeted therapy.

Notch signaling has been demonstrated as an important mediator in TGFa-induced changes in epithelial differentiation during pancreatic tumorigenesis 76 . The interaction of Notch ligands with their receptors promotes a $\gamma$-secretase-dependent cleavage of the Notch receptor and release of the Notch intracellular domain 59. Plentz et al tested the efficacy of a $\gamma$-secretase inhibitor (GSI), which blocks the Notch signaling, in pancreatic cancer cell lines and a genetically engineered mouse model of pancreatic cancer 59 . Their results indicate that targeting Notch pathway offers therapeutic benefit in pancreatic cancer treatment. Wang and colleagues also demonstrated that downregulation of Notch signaling was associated with decreased invasion of gemcitabine-resistant cells and led to partial reversal of the EMT phenotype 54. These findings present strong in vitro and in vivo evidence for a novel strategy targeting Notch signaling pathway in pancreatic cancer therapy.

EMT is a critical process in pancreatic cancer progression, and has a wide connection with many other drug-resistance mechanisms: (a) aberrant gene expression, such as PI3K, snail, Zeb1, and TGF- $\beta$; (b) deregulated signaling pathways including the PI3K/Akt, Notch, and NF- $\mathrm{kB}$ signaling pathways; (c) cancer stem-like cells; and (d) hypoxia environment. EMT is thought to be one of the major causes of the formation of the dense desmoplastic 
microenvironment with low density of vasculature, which makes it very difficult for any chemotherapy drugs to penetrate. Targeting EMT-related pathways is likely to generate more favorable clinical outcomes in pancreatic cancer therapy. In a recent study, Li et al compared the expression of microRNAs (miRNAs) between gemcitabine-sensitive and gemcitabine-resistant pancreatic cancer cells and found that the expression of miR-200b, miR-200c, let-7b, let-7c, let-7d, and let-7e was significantly downregulated in gemcitabineresistant cells, which showed EMT characteristics with decreased expression of epithelial marker E-cadherin, and increased expression of mesenchymal markers such as vimentin and ZEB1. Overexpression of miR-200 by transfection or treatment of gemcitabine-resistant cells with either DIM or isoflavone resulted in the downregulation of ZEB1, slug, and vimentin, which was consistent with morphologic reversal of EMT phenotype. These results indicate the importance of the EMT pathway in pancreatic cancer development, and suggest new therapeutic strategies using DIM and isoflavone, and possible involvement of microRNAs in the regulation of EMT phenotype in pancreatic cancer 77.

Targeting the sphingolipid metabolism for improving tumor chemosensitivity has recently emerged as a promising strategy. Sphingosine kinase-1 (SphK1), a tumor-associated protein overexpressed in many cancers, favors survival through sphingosine-1-phosphate (S1P) production, and inhibitors of SphK1 are used in ongoing clinical trials to sensitize epithelial ovarian and prostate cancer cells to various chemotherapeutic drugs. The cellular ceramide/ $\mathrm{S} 1 \mathrm{P}$ ratio is a critical biosensor for predicting pancreatic cancer cell sensitivity to gemcitabine. A low level of the ceramide/S1P ratio, associated with a high SphK1 activity, correlates with a robust intrinsic pancreatic cancer cell chemoresistance toward gemcitabine. Strikingly, increasing the ceramide/S1P ratio by using pharmacologic (SphK1 inhibitor or ceramide analogue) or small interfering RNA-based approaches to up-regulate intracellular ceramide levels or reduce SphK1 activity sensitized pancreatic cancer cells to gemcitabine. Conversely, decreasing the ceramide/S1P ratio by upregulating SphK1 activity promoted gemcitabine resistance in these cells. Development of novel pharmacologic strategies targeting sphingolipid metabolism might therefore represent an interesting approach, when combined with gemcitabine, to defeat pancreatic cancer chemoresistance to this drug. 39

Survivin is known to play an important role in resistance to radiation and chemotherapy in pancreatic cancer and targeting survivin could be a new therapeutic strategy. Liu et al showed that silencing of survivin expression with siRNA caused spontaneous apoptosis in human pancreatic cancer cell lines. Furthermore, the decrease of survivin can enhance the chemosensitivity of pancreatic cancer cells to gemcitabine 40 . The level of Plk-1 protein was also found to be correlated with gemcitabine resistance, and overexpression of Plk-1 reduced sensitivity to gemcitabine in tumor cells. Yu et al transfected pancreatic cancer cells with Plk-1 siRNA and they found that downregulation of Plk-1 caused cell cycle arrest at G2/M phase, enhanced the chemosensitivity to gemcitabine, and dramatically decreased cell viability and increased cellular apoptosis 37 .

\section{Other strategies in overcoming drug resistance in pancreatic cancer}

As discussed above, hypoxia within pancreatic cancer due to insufficient vascularization, and the presence of stroma cells confer further resistance to both chemotherapy and radiotherapy. Thus, strategies to improve the sensitivity of chemotherapy should include anti-hypoxia and anti-stroma cells, and should increase the local concentration of chemotherapy drugs. Many studies have demonstrated promising results of intra-arterial chemotherapy via the femoral artery under digital subtraction angiography with the tip inserted directly into the hepatic artery and the superior mesenteric artery for pancreatic cancer treatment 78, 79. Intra-arterial chemotherapy may lead to higher drug concentration in the pancreas compared to systemic chemotherapy, and could improve the therapeutic effect. In a recent study, Olive et al used IPI-926, a drug that depletes tumor-associated 
stromal tissue by inhibition of the Hedgehog signaling pathway, to test whether the delivery and efficacy of gemcitabine could be improved in a mouse model 63. This new therapy produced a transient increase in intratumoral vascular density and intratumoral concentration of gemcitabine, and led to transient stabilization of this disease, suggesting that impaired drug delivery may be an important contributor to drug resistance in pancreatic cancer. Eradiation of stem cells is another strategy to consider in order to improve pancreatic cancer treatment. Many obstacles still exist for this strategy including identifying more accurate surface markers for cancer stem cells, and developing more specific methods to eradicate cancer stem cells. It is likely that the combinational therapy that targets both the pancreatic cancer stem cells and the differentiated bulk population of pancreatic cancer cells will be most potent in treating pancreatic cancer patients by reducing tumor mass and preventing relapse 64 .

\section{Clinical Information}

Continuous efforts have been made to overcome drug resistance in clinical trials although most of them failed. Gemcitabine and other chemotherapy drugs such as Oxaliplatin have been used in combination with small molecule inhibitors, dominant negative constructs, gene therapy, and monoclonal antibodies, etc. In a phase II trial, Reni and colleagues demonstrated the potential to treat gemcitabine-resistant metastatic pancreatic cancer using a combination treatment of Raltitrexid, a thymidylate synthase inhibitor that polyaglutamates in the cell to extend intracellular retention, and Oxaliplatin, a platinum analogue that forms interstrand DNA adducts capable of overcoming resistance mechanisms 80. Another trial showed that oxaliplatin in combination with gemcitabine is a well-tolerated treatment that may provide a benefit to patients with advanced gemcitabine-refractory pancreatic cancer 81. Chawla et al recently showed in a phase I/II trial that Rexin-G, a nonreplicative-targeted retroviral gene therapy vector bearing a cytocidal dominant negative cyclin G1 construct, is safe, well-tolerated, and may help control tumor growth and prolong survival in gemcitabine-resistant pancreatic cancer patients 82 . An ongoing phase II study (NCT00408564) is studying whether the monoclonal antibody cetuximab will help overcome drug resistance by giving cetuximab together with chemotherapeutic agents gemcitabine, capecitabine, and oxaliplatin followed by surgery or external beam radiation therapy to patients with locally advanced, non-metastatic pancreatic cancer. CO-1.01, a newly developed gemcitabine derivative, works independently of the intracellular uptake protein hENT1 and may benefit patients with low hENT1 expression who failed to respond to gemcitabine. A phase II study (NCT01233375) is currently recruiting participants to determine the safety and efficacy of CO-1.01 as an alternative to gemcitabine that is unaffected by hENT1 chemosensitivity. Targeting pancreatic cancer stem cells is a viable and potent approach to overcome drug resistance. A multicenter phase II study (NCT01088815) is testing the efficacy of the hedgehog signaling pathway inhibitor GDC-0449 in metastatic pancreatic cancer, and a pilot study (NCT01195415) is testing the same drug with a specific focus on its ability to target the hedgehog pathway in pancreatic cancer stem cells in combination with gemcitabine treatment. A phase I/II trial (NCT01098344) targeting the Notch pathway using the Notch inhibitor MK-0752 in patients with stage IV pancreatic cancer is also currently recruiting. The new therapies currently being developed such those we discussed earlier, including inhibiting aberrant gene expression, deregulated signal transduction pathways, and tumor microenvironment and cancer stem cells may provide new hope for overcoming drug resistance in pancreatic cancer. 


\section{Summary}

Over a 30-year period, there were minor changes in the stage at diagnosis, resection for cure and prognosis of pancreatic cancers, although there were some improvements in the diagnostic modalities. Pancreatic cancer still represents a major challenge and is the most deadly cancer 83 . Drug resistance is a key factor that limits the choice of therapies and significantly decreases the efficacy of any chemotherapy drugs for pancreatic cancer. New breakthroughs to overcome chemoresistance will improve the clinical outcomes of pancreatic cancer. Many mechanisms contribute to drug resistance in pancreatic cancer including aberrant gene expression, deregulated signaling pathways, hypoxia, tumor microenvironment, and the presence of cancer stem cells. Understanding those mechanisms will help to develop specific strategies in overcoming drug resistance and improve the efficacy of pancreatic cancer treatment.

\section{Expert Opinion}

For many years, drug resistance (intrinsic and acquired) has been a major problem for pancreatic cancer treatment. Although new drugs and therapeutic strategies have been constantly developed, most of them are ineffective in clinical trials because of the poor penetration of those drugs into the pancreas, and the unresponsiveness of pancreatic tumors to the drugs, which is largely due to the drug resistance property of pancreatic cancer. How to increase drug delivery efficiency and decrease drug resistance becomes the priority in pancreatic cancer treatment, and will also benefit the treatment of other cancers and other diseases. Understanding the molecular and cellular basis of drug resistance in pancreatic cancer will lead to development of novel therapeutic strategies which have potential to increase the efficacy of current treatments of a wide variety of human cancers with drug resistance characteristics.

To date there are a great number of candidate genes and pathways identified for drug resistance in pancreatic cancer, which will serve as potential therapeutic targets. However, many of them are not specific in pancreatic cancer, and the clinical impact of those targets is questionable. In the next decade, continued efforts are needed to find more specific and potent targeted therapies to overcome the drug resistance in pancreatic cancer. It is critical that the drug-resistance gene signature should be established in the future, which will be used to develop new therapies that can combine the anti-drug resistance with conventional therapy in pancreatic cancer. Patients can then be grouped based on their gene profiling and receive personalized medicine.

Such a program requires both a strong basic science research effort on targeted genes as well as an efficient delivery system. In pancreatic cancer, quite a few molecules have been identified as potential therapeutic markers, such as CEACAM-1, CEACAM-6, S100P, ataxia-telangiectasia group D complementing gene (ATDC), PDX-1, mesothelin, and ZIP4 84-92. Our recent studies indicate that ZIP4 is overexpressed in human pancreatic cancer cells and tumor tissues, and silencing of ZIP4 caused decreased pancreatic cancer growth and increased survival rate in a mouse model, suggesting that ZIP4 is a novel therapeutic target in pancreatic cancer 88,93 . Blocking the expression and activity of one or more above mentioned molecules may not only inhibit pancreatic cancer growth, but also further sensitize pancreatic cancer cells to chemotherapy drugs. Developing efficient targeted drug delivery system is also important in pancreatic cancer treatment. Using liposomes, nanoparticles, or carbon nanotubes to deliver chemotherapy drugs or other therapeutic agents has been a success in recent preclinical trials. Conjugation with ligand or antibody for targeted delivery may further increase the efficacy of the delivery to reach the designated organs, such as pancreas 94 . 
In addition to identifying new targets and better delivery systems, finding novel cytotoxic agents and complimentary treatment can also improve the reversal of drug resistance in pancreatic cancer. Angelova et al recently combined gemcitabine and oncolytic virus (H-1PV) treatment in a pancreatic cancer model. The in vitro results indicated that the combination therapy showed synergistic cytotoxic effects in drug-resistant cells which are sensitive to virus infection 95. In another study, Kindler et al treated patients with advanced pancreatic cancer after gemcitabine therapy with arsenic trioxide, which has cytotoxic antiproliferative effects in tumor cells, in a multicenter phase II clinical trial 96. Despite the promising results in vitro, the arsenic trioxide has no activity in pancreatic cancer patients. Clearly, the discovery of novel potent therapeutic agents is urgently needed. New formulation of drugs is also important for anti-drug resistance. Graeser et al synthesized liposomal gemcitabine (GemLip) and compared the efficacy of Gemlip with gemcitabine in vivo. GemLip is an effective new formulation of gemcitabine, which showed targeting, protective features and antitumor effects 97 . Therefore, control-released drugs and novel therapeutic agents carrying special molecular targets represent a new therapeutic strategy to overcome the drug resistance in pancreatic cancer.

Article highlight box:

- Pancreatic cancer has the worst survival rate of all cancers. The current standard care for metastatic pancreatic cancer is gemcitabine, however, the success of this treatment is poor, and overall survival has not improved for decades.

- Drug resistance (both intrinsic and acquired) is thought to be a major reason for the limited benefit of most pancreatic cancer therapies.

- Mechanisms of drug resistance in pancreatic cancer include aberrant gene expression, mutations, deregulation of key signaling pathways, epithelialmesenchymal transition (EMT) and the presence of stroma cells, highly resistant cells and stem cells.

- Understanding the molecular and cellular basis of drug resistance in pancreatic cancer will lead to the development of novel therapeutic strategies which have potential to sensitize pancreatic cancer to chemotherapy, and increase the efficacy of current treatments in pancreatic cancer.

\section{Acknowledgments}

We thank Dr. Rosa Hwang for valuable suggestions.

This work was supported in part by the National Institutes of Health (NIH) grant R21CA133604, R01CA138701, the William and Ella Owens Medical Research Foundation (M. Li), and the National Science Foundation of China grant 30701007 (J. Long).

\section{References}

1. Jemal A, Siegel R, Ward E, et al. Cancer statistics, 2009. CA Cancer J Clin. Jul-Aug;2009 59(4): 225-49. [PubMed: 19474385]

2. Landis SH, Murray T, Bolden S, et al. Cancer statistics, 1998. CA Cancer J Clin. Jan-Feb;1998 48(1):6-29. [PubMed: 9449931]

3. Torrisani J, Buscail L. Molecular pathways of pancreatic carcinogenesis. Ann Pathol. Oct; 2002 22(5):349-55. [PubMed: 12483152]

4. Warshaw AL, Fernandez-del Castillo C. Pancreatic carcinoma. N Engl J Med. Feb 13; 1992 326(7): 455-65. [PubMed: 1732772] 
5. Fisher WE, Berger DH. Angiogenesis and antiangiogenic strategies in pancreatic cancer. Int J Gastrointest Cancer. 2003; 33(1):79-88. [PubMed: 12909740]

6. Day JD, Digiuseppe JA, Yeo C, et al. Immunohistochemical evaluation of HER-2/neu expression in pancreatic adenocarcinoma and pancreatic intraepithelial neoplasms. Hum Pathol. Feb; 1996 27(2): 119-24. [PubMed: 8617452]

7. Hruban RH, van Mansfeld AD, Offerhaus GJ, et al. K-ras oncogene activation in adenocarcinoma of the human pancreas. A study of 82 carcinomas using a combination of mutant-enriched polymerase chain reaction analysis and allele-specific oligonucleotide hybridization. Am J Pathol. Aug; 1993 143(2):545-54. [PubMed: 8342602]

8. DiGiuseppe JA, Hruban RH, Goodman SN, et al. Overexpression of p53 protein in adenocarcinoma of the pancreas. Am J Clin Pathol. Jun; 1994 101(6):684-8. [PubMed: 8209852]

9. Sturm PD, Hruban RH, Ramsoekh TB, et al. The potential diagnostic use of K-ras codon 12 and p53 alterations in brush cytology from the pancreatic head region. J Pathol. Nov; 1998 186(3):247-53. [PubMed: 10211112]

10. van Heek T, Rader AE, Offerhaus GJ, et al. K-ras, p53, and DPC4 (MAD4) alterations in fineneedle aspirates of the pancreas: a molecular panel correlates with and supplements cytologic diagnosis. Am J Clin Pathol. May; 2002 117(5):755-65. [PubMed: 12090425]

11. Burris HA 3rd, Moore MJ, Andersen J, et al. Improvements in survival and clinical benefit with gemcitabine as first-line therapy for patients with advanced pancreas cancer: a randomized trial. J Clin Oncol. Jun; 1997 15(6):2403-13. [PubMed: 9196156] **First study showing that Gemcitabine is the first-line therapy with advanced pancreatic cancer.

12. Berlin JD, Catalano P, Thomas JP, et al. Phase III study of gemcitabine in combination with fluorouracil versus gemcitabine alone in patients with advanced pancreatic carcinoma: Eastern Cooperative Oncology Group Trial E2297. J Clin Oncol. Aug 1; 2002 20(15):3270-5. [PubMed: 12149301]

13. Heinemann V, Quietzsch D, Gieseler F, et al. Randomized phase III trial of gemcitabine plus cisplatin compared with gemcitabine alone in advanced pancreatic cancer. J Clin Oncol. Aug 20; 2006 24(24):3946-52. [PubMed: 16921047]

14. Louvet C, Labianca R, Hammel P, et al. Gemcitabine in combination with oxaliplatin compared with gemcitabine alone in locally advanced or metastatic pancreatic cancer: results of a GERCOR and GISCAD phase III trial. J Clin Oncol. May 20; 2005 23(15):3509-16. [PubMed: 15908661]

15. Herrmann R, Bodoky G, Ruhstaller T, et al. Gemcitabine plus capecitabine compared with gemcitabine alone in advanced pancreatic cancer: a randomized, multicenter, phase III trial of the Swiss Group for Clinical Cancer Research and the Central European Cooperative Oncology Group. J Clin Oncol. Jun 1; 2007 25(16):2212-7. [PubMed: 17538165]

16. Moore MJ, Goldstein D, Hamm J, et al. Erlotinib plus gemcitabine compared with gemcitabine alone in patients with advanced pancreatic cancer: a phase III trial of the National Cancer Institute of Canada Clinical Trials Group. J Clin Oncol. May 20; 2007 25(15):1960-6. [PubMed: 17452677]

17. Cascinu S, Verdecchia L, Valeri N, et al. New target therapies in advanced pancreatic cancer. Ann Oncol. May; 2006 17(Suppl 5):v148-52. [PubMed: 16807445]

18. Kindler HL, Friberg G, Singh DA, et al. Phase II trial of bevacizumab plus gemcitabine in patients with advanced pancreatic cancer. J Clin Oncol. Nov 1; 2005 23(31):8033-40. [PubMed: 16258101]

19. Tredan O, Galmarini CM, Patel K, et al. Drug resistance and the solid tumor microenvironment. J Natl Cancer Inst. Oct 3; 2007 99(19):1441-54. [PubMed: 17895480]

20. Bisht S, Feldmann G, Koorstra JB, et al. In vivo characterization of a polymeric nanoparticle platform with potential oral drug delivery capabilities. Mol Cancer Ther. Dec; 2008 7(12):387888. [PubMed: 19074860]

21. Zhou J, Liu M, Aneja R, et al. Reversal of P-glycoprotein-mediated multidrug resistance in cancer cells by the c-Jun NH2-terminal kinase. Cancer Res. Jan 1; 2006 66(1):445-52. [PubMed: 16397260]

22. Higgins CF. ABC transporters: from microorganisms to man. Annu Rev Cell Biol. 1992; 8:67113. [PubMed: 1282354] *The importance of ABC transporters in cancer. 
23. Gottesman MM, Ambudkar SV. Overview: ABC transporters and human disease. J Bioenerg Biomembr. Dec; 2001 33(6):453-8. [PubMed: 11804186]

24. Lage H. An overview of cancer multidrug resistance: a still unsolved problem. Cell Mol Life Sci. Oct; 2008 65(20):3145-67. [PubMed: 18581055]

25. Sharom FJ, Liu R, Qu Q, et al. Exploring the structure and function of the P-glycoprotein multidrug transporter using fluorescence spectroscopic tools. Semin Cell Dev Biol. Jun; 2001 12(3):257-65. [PubMed: 11428918]

26. Dean M, Hamon Y, Chimini G. The human ATP-binding cassette (ABC) transporter superfamily. J Lipid Res. Jul; 2001 42(7):1007-17. [PubMed: 11441126]

27. Kruh GD, Belinsky MG. The MRP family of drug efflux pumps. Oncogene. Oct 20; 2003 22(47): 7537-52. [PubMed: 14576857]

28. Konig J, Hartel M, Nies AT, et al. Expression and localization of human multidrug resistance protein (ABCC) family members in pancreatic carcinoma. Int J Cancer. Jun 20; 2005 115(3):359_ 67. [PubMed: 15688370]

29. Kool M, van der Linden M, de Haas M, et al. MRP3, an organic anion transporter able to transport anti-cancer drugs. Proc Natl Acad Sci U S A. Jun 8; 1999 96(12):6914-9. [PubMed: 10359813]

30. Hagmann W, Jesnowski R, Lohr JM. Interdependence of gemcitabine treatment, transporter expression, and resistance in human pancreatic carcinoma cells. Neoplasia. Sep; 12(9):740-7. [PubMed: 20824050]

31. Nambaru PK, Hubner T, Kock K, et al. Drug efflux transporter multidrug resistance-associated protein 5 affects sensitivity of pancreatic cancer cell lines to the nucleoside anticancer drug 5fluorouracil. Drug Metab Dispos. Jan; 39(1):132-9. [PubMed: 20930123]

32. Andersson R, Aho U, Nilsson BI, et al. Gemcitabine chemoresistance in pancreatic cancer: Molecular mechanisms and potential solutions. Scand J Gastroenterol. Feb $13.2009: 1-5$. [PubMed: 19707944]

33. Mackey JR, Mani RS, Selner M, et al. Functional nucleoside transporters are required for gemcitabine influx and manifestation of toxicity in cancer cell lines. Cancer Res. Oct 1; 1998 58(19):4349-57. [PubMed: 9766663] *Gemcitabine and its receptor hENT1.

34. Bergman AM, Pinedo HM, Peters GJ. Determinants of resistance to $2^{\prime}, 2^{\prime}$-difluorodeoxycytidine (gemcitabine). Drug Resist Updat. Feb; 2002 5(1):19-33. [PubMed: 12127861]

35. Kim MP, Gallick GE. Gemcitabine resistance in pancreatic cancer: picking the key players. Clin Cancer Res. Mar 1; 2008 14(5):1284-5. [PubMed: 18316544]

36. Holzer AK, Varki NM, Le QT, et al. Expression of the human copper influx transporter 1 in normal and malignant human tissues. J Histochem Cytochem. Sep; 2006 54(9):1041-9. [PubMed: 16709730]

37. Yu C, Zhang X, Sun G, et al. RNA interference-mediated silencing of the polo-like kinase 1 gene enhances chemosensitivity to gemcitabine in pancreatic adenocarcinoma cells. J Cell Mol Med. Dec; 2008 12(6A):2334-49. [PubMed: 18266952]

38. Yin T, Wang C, Liu T, et al. Expression of snail in pancreatic cancer promotes metastasis and chemoresistance. J Surg Res. Aug; 2007 141(2):196-203. [PubMed: 17583745]

39. Guillermet-Guibert J, Davenne L, Pchejetski D, et al. Targeting the sphingolipid metabolism to defeat pancreatic cancer cell resistance to the chemotherapeutic gemcitabine drug. Mol Cancer Ther. Apr; 2009 8(4):809-20. [PubMed: 19372554]

40. Liu WS, Yan HJ, Qin RY, et al. siRNA directed against survivin enhances pancreatic cancer cell gemcitabine chemosensitivity. Dig Dis Sci. Jan; 2009 54(1):89-96. [PubMed: 18594980]

41. Duxbury MS, Ito H, Zinner MJ, et al. siRNA directed against c-Src enhances pancreatic adenocarcinoma cell gemcitabine chemosensitivity. J Am Coll Surg. Jun; 2004 198(6):953-9. [PubMed: 15194078]

42. Tutt AN, Lord CJ, McCabe N, et al. Exploiting the DNA repair defect in BRCA mutant cells in the design of new therapeutic strategies for cancer. Cold Spring Harb Symp Quant Biol. 2005; 70:139-48. [PubMed: 16869747] *Gene mutations and drug resistance.

43. Farmer H, McCabe N, Lord CJ, et al. Targeting the DNA repair defect in BRCA mutant cells as a therapeutic strategy. Nature. Apr 14; 2005 434(7035):917-21. [PubMed: 15829967] 
44. Bryant HE, Schultz N, Thomas HD, et al. Specific killing of BRCA2-deficient tumours with inhibitors of poly(ADP-ribose) polymerase. Nature. Apr 14; 2005 434(7035):913-7. [PubMed: 15829966]

45. Edwards SL, Brough R, Lord CJ, et al. Resistance to therapy caused by intragenic deletion in BRCA2. Nature. Feb 28; 2008 451(7182):1111-5. [PubMed: 18264088]

46. Ashworth A. Drug resistance caused by reversion mutation. Cancer Res. Dec 15; 2008 68(24): 10021-3. [PubMed: 19074863]

47. Ghobrial IM, Witzig TE, Adjei AA. Targeting apoptosis pathways in cancer therapy. CA Cancer J Clin. May-Jun;2005 55(3):178-94. [PubMed: 15890640]

48. Banerjee S, Wang Z, Kong D, et al. 3,3'-Diindolylmethane enhances chemosensitivity of multiple chemotherapeutic agents in pancreatic cancer. Cancer Res. Jul 1; 2009 69(13):5592-600. [PubMed: 19531648] *NF- $\mathrm{kB}$ pathway and drug resistance.

49. Bharti AC, Aggarwal BB. Nuclear factor-kappa B and cancer: its role in prevention and therapy. Biochem Pharmacol. Sep; 2002 64(5-6):883-8. [PubMed: 12213582] *NF- B pathway and drug resistance.

50. Liau SS, Jazag A, Whang EE. HMGA1 is a determinant of cellular invasiveness and in vivo metastatic potential in pancreatic adenocarcinoma. Cancer Res. Dec 15; 2006 66(24):11613-22. [PubMed: 17178855]

51. Ng SSW, Tsao MS, Chow S, et al. Inhibition of phosphatidylinositide 3-kinase enhances gemcitabine-induced apoptosis in human pancreatic cancer cells. Cancer Res. Oct 1; 2000 60(19): 5451-5. [PubMed: 11034087]

52. Sourbier C, Lindner V, Lang H, et al. The phosphoinositide 3-kinase/Akt pathway: a new target in human renal cell carcinoma therapy. Cancer Res. May 15; 2006 66(10):5130-42. [PubMed: 16707436]

53. Ellenrieder V, Hendler SF, Boeck W, et al. Transforming growth factor beta1 treatment leads to an epithelial-mesenchymal transdifferentiation of pancreatic cancer cells requiring extracellular signal-regulated kinase 2 activation. Cancer Res. May 15; 2001 61(10):4222-8. [PubMed: 11358848] *EMT and drug resistance.

54. Wang Z, Li Y, Kong D, et al. Acquisition of epithelial-mesenchymal transition phenotype of gemcitabine-resistant pancreatic cancer cells is linked with activation of the notch signaling pathway. Cancer Res. Mar 15; 2009 69(6):2400-7. [PubMed: 19276344]

55. Min C, Eddy SF, Sherr DH, et al. NF-kappaB and epithelial to mesenchymal transition of cancer. J Cell Biochem. Jun 1; 2008 104(3):733-44. [PubMed: 18253935]

56. Sahlgren C, Gustafsson MV, Jin S, et al. Notch signaling mediates hypoxia-induced tumor cell migration and invasion. Proc Natl Acad Sci U S A. Apr 29; 2008 105(17):6392-7. [PubMed: 18427106]

57. Shah AN, Summy JM, Zhang J, et al. Development and characterization of gemcitabine-resistant pancreatic tumor cells. Ann Surg Oncol. Dec; 2007 14(12):3629-37. [PubMed: 17909916]

58. Arumugam T, Ramachandran V, Fournier KF, et al. Epithelial to mesenchymal transition contributes to drug resistance in pancreatic cancer. Cancer Res. Jul 15; 2009 69(14):5820-8. [PubMed: 19584296]

59. Plentz R, Park JS, Rhim AD, et al. Inhibition of gamma-secretase activity inhibits tumor progression in a mouse model of pancreatic ductal adenocarcinoma. Gastroenterology. May; 2009 136(5):1741-9 e6. [PubMed: 19208345]

60. Masamune A, Kikuta K, Watanabe T, et al. Fibrinogen induces cytokine and collagen production in pancreatic stellate cells. Gut. Apr; 2009 58(4):550-9. [PubMed: 19052021]

61. Hwang RF, Moore T, Arumugam T, et al. Cancer-associated stromal fibroblasts promote pancreatic tumor progression. Cancer Res. Feb 1; 2008 68(3):918-26. [PubMed: 18245495] * Stromal cells and drug resistance.

62. Vonlaufen A, Phillips PA, Xu Z, et al. Pancreatic stellate cells and pancreatic cancer cells: an unholy alliance. Cancer Res. Oct 1; 2008 68(19):7707-10. [PubMed: 18829522]

63. Olive KP, Jacobetz MA, Davidson CJ, et al. Inhibition of Hedgehog signaling enhances delivery of chemotherapy in a mouse model of pancreatic cancer. Science. Jun 12; 2009 324(5933):1457-61. [PubMed: 19460966] *Hedgehog signal and drug resistance in pancreatic cancer. 
64. Simeone DM. Pancreatic cancer stem cells: implications for the treatment of pancreatic cancer. Clin Cancer Res. Sep 15; 2008 14(18):5646-8. [PubMed: 18794070]

65. Lee CJ, Dosch J, Simeone DM. Pancreatic cancer stem cells. J Clin Oncol. Jun 10; 2008 26(17): 2806-12. [PubMed: 18539958]

66. Li C, Heidt DG, Dalerba P, et al. Identification of pancreatic cancer stem cells. Cancer Res. Feb 1; 2007 67(3):1030-7. [PubMed: 17283135] **Stem cells and pancreatic cancer.

67. Hermann PC, Huber SL, Herrler T, et al. Distinct populations of cancer stem cells determine tumor growth and metastatic activity in human pancreatic cancer. Cell Stem Cell. Sep 13; 2007 1(3):31323. [PubMed: 18371365]

68. O'Brien CA, Pollett A, Gallinger S, et al. A human colon cancer cell capable of initiating tumour growth in immunodeficient mice. Nature. Jan 4; 2007 445(7123):106-10. [PubMed: 17122772]

69. Singh SK, Hawkins C, Clarke ID, et al. Identification of human brain tumour initiating cells. Nature. Nov 18; 2004 432(7015):396-401. [PubMed: 15549107]

70. Dembinski JL, Krauss S. Characterization and functional analysis of a slow cycling stem cell-like subpopulation in pancreas adenocarcinoma. Clin Exp Metastasis. 2009; 26(7):611-23. [PubMed: 19421880]

71. Jimeno A, Feldmann G, Suarez-Gauthier A, et al. A direct pancreatic cancer xenograft model as a platform for cancer stem cell therapeutic development. Mol Cancer Ther. Feb; 2009 8(2):310-4. [PubMed: 19174553]

72. Hoffmann K, Marten A, Lindel K, et al. Major combined electrolyte deficiency during therapy with low-dose Cisplatin, 5-Fluorouracil and Interferon alpha: report on several cases and review of the literature [ISRCTN62866759]. BMC Cancer. May 10.2006 6(1):128. [PubMed: 16686958]

73. Pan X, Arumugam T, Yamamoto T, et al. Nuclear factor-kappaB p65/relA silencing induces apoptosis and increases gemcitabine effectiveness in a subset of pancreatic cancer cells. Clin Cancer Res. Dec 15; 2008 14(24):8143-51. [PubMed: 19088029]

74. Fahy BN, Schlieman MG, Virudachalam S, et al. Inhibition of AKT abrogates chemotherapyinduced NF-kappaB survival mechanisms: implications for therapy in pancreatic cancer. J Am Coll Surg. Apr; 2004 198(4):591-9. [PubMed: 15051014]

75. Schlieman MG, Fahy BN, Ramsamooj R, et al. Incidence, mechanism and prognostic value of activated AKT in pancreas cancer. Br J Cancer. Dec 1; 2003 89(11):2110-5. [PubMed: 14647146]

76. Miyamoto Y, Maitra A, Ghosh B, et al. Notch mediates TGF alpha-induced changes in epithelial differentiation during pancreatic tumorigenesis. Cancer Cell. Jun; 2003 3(6):565-76. [PubMed: 12842085]

77. Li Y, Vandenboom TG 2nd, Kong D, et al. Up-regulation of miR-200 and let-7 by Natural Agents Leads to the Reversal of Epithelial-to-Mesenchymal Transition in Gemcitabine-Resistant Pancreatic Cancer Cells. Cancer Res. Aug 4.2009*MicroRNA and drug resistance.

78. Jin C, Yao L, Long J, et al. Effect of multiple-phase regional intra-arterial infusion chemotherapy on patients with resectable pancreatic head adenocarcinoma. Chin Med J (Engl). Feb 5; 2009 122(3):284-90. [PubMed: 19236805]

79. Yang F, Long J, Fu DL, et al. Aberrant hepatic artery in patients undergoing pancreaticoduodenectomy. Pancreatology. 2008; 8(1):50-4. [PubMed: 18230918]

80. Reni M, Pasetto L, Aprile G, et al. Raltitrexed-eloxatin salvage chemotherapy in gemcitabineresistant metastatic pancreatic cancer. Br J Cancer. Mar 27; 2006 94(6):785-91. [PubMed: 16508631]

81. Demols A, Peeters M, Polus M, et al. Gemcitabine and oxaliplatin (GEMOX) in gemcitabine refractory advanced pancreatic adenocarcinoma: a phase II study. Br J Cancer. Feb 27; 2006 94(4): 481-5. [PubMed: 16434988]

82. Chawla SP, Chua VS, Fernandez L, et al. Advanced phase I/II studies of targeted gene delivery in vivo: intravenous Rexin-G for gemcitabine-resistant metastatic pancreatic cancer. Mol Ther. Feb; 18(2):435-41. [PubMed: 19826403]

83. David M, Lepage C, Jouve JL, et al. Management and prognosis of pancreatic cancer over a 30year period. Br J Cancer. Jul 21; 2009 101(2):215-8. [PubMed: 19568238] 
84. Wang L, Heidt DG, Lee CJ, et al. Oncogenic function of ATDC in pancreatic cancer through Wnt pathway activation and beta-catenin stabilization. Cancer Cell. Mar 3; 2009 15(3):207-19. [PubMed: 19249679]

85. Duxbury MS, Matros E, Ito H, et al. Systemic siRNA-mediated gene silencing: a new approach to targeted therapy of cancer. Ann Surg. Oct; 2004 240(4):667-74. discussion 75-6. [PubMed: 15383794]

86. Duxbury MS, Ito H, Zinner MJ, et al. CEACAM6 gene silencing impairs anoikis resistance and in vivo metastatic ability of pancreatic adenocarcinoma cells. Oncogene. Jan 15; 2004 23(2):465-73. [PubMed: 14724575]

87. Arumugam T, Simeone DM, Van Golen K, et al. S100P promotes pancreatic cancer growth, survival, and invasion. Clin Cancer Res. Aug 1; 2005 11(15):5356-64. [PubMed: 16061848]

88. Li M, Zhang Y, Liu Z, et al. Aberrant expression of zinc transporter ZIP4 (SLC39A4) significantly contributes to human pancreatic cancer pathogenesis and progression. Proc Natl Acad Sci U S A. Nov 20; 2007 104(47):18636-41. [PubMed: 18003899] **ZIP4 pathway and pancreatic cancer.

89. Li M, Bharadwaj U, Zhang R, et al. Mesothelin is a malignant factor and therapeutic vaccine target for pancreatic cancer. Mol Cancer Ther. Feb; 2008 7(2):286-96. [PubMed: 18281514]

90. Hucl T, Brody JR, Gallmeier E, et al. High cancer-specific expression of mesothelin (MSLN) is attributable to an upstream enhancer containing a transcription enhancer factor dependent MCAT motif. Cancer Res. Oct 1; 2007 67(19):9055-65. [PubMed: 17909009]

91. Hassan R, Laszik ZG, Lerner M, et al. Mesothelin is overexpressed in pancreaticobiliary adenocarcinomas but not in normal pancreas and chronic pancreatitis. Am J Clin Pathol. Dec; 2005 124(6):838-45. [PubMed: 16416732]

92. Liu S, Ballian N, Belaguli NS, et al. PDX-1 acts as a potential molecular target for treatment of human pancreatic cancer. Pancreas. Aug; 2008 37(2):210-20. [PubMed: 18665085]

93. Li M, Zhang Y, Bharadwaj U, et al. Down-regulation of ZIP4 by RNA interference inhibits pancreatic cancer growth and increases the survival of nude mice with pancreatic cancer xenografts. Clin Cancer Res. Oct 1; 2009 15(19):5993-6001. [PubMed: 19755388]

94. Yu X, Zhang Y, Chen C, et al. Targeted drug delivery in pancreatic cancer. Biochim Biophys Acta. Oct 22.2009

95. Angelova AL, Aprahamian M, Grekova SP, et al. Improvement of gemcitabine-based therapy of pancreatic carcinoma by means of oncolytic parvovirus H-1PV. Clin Cancer Res. Jan 15; 2009 15(2):511-9. [PubMed: 19147756]

96. Kindler HL, Aklilu M, Nattam S, et al. Arsenic trioxide in patients with adenocarcinoma of the pancreas refractory to gemcitabine: a phase II trial of the University of Chicago Phase II Consortium. Am J Clin Oncol. Dec; 2008 31(6):553-6. [PubMed: 19060586]

97. Graeser R, Bornmann C, Esser N, et al. Antimetastatic effects of liposomal gemcitabine and empty liposomes in an orthotopic mouse model of pancreatic cancer. Pancreas. Apr; 2009 38(3):330-7. [PubMed: 19142174] 


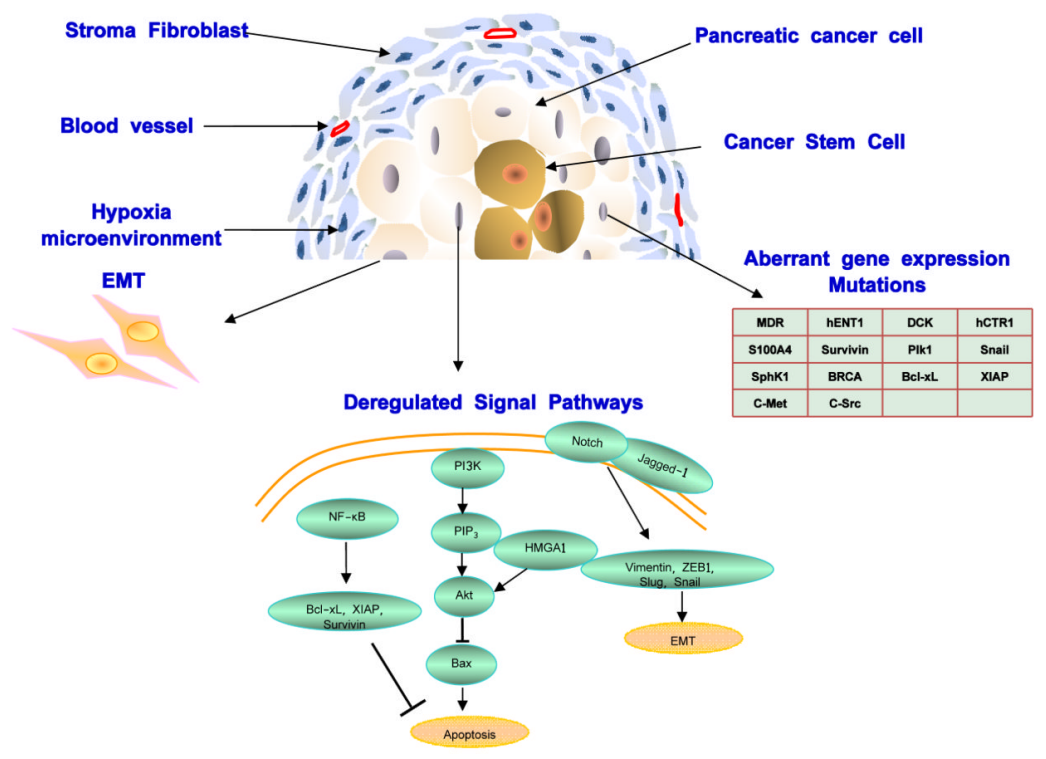

Figure 1. Drug resistance pathways in pancreatic cancer

Drug resistance in pancreatic cancer is caused by various mechanisms including aberrant gene expression, mutations, deregulation of key signaling pathways (such as NF- $\kappa \mathrm{B}$, Notch, Akt, and apoptosis pathways), epithelial-mesenchymal transition (EMT) and the presence of highly resistant cells and stem cells. Each of those mechanisms contributes to drug resistance in pancreatic cancer from different aspects, and suggests different therapeutic targets. A few representative drug resistance pathways such as NF- $\kappa B$, Notch, Akt, and apoptosis pathways are shown. 